\title{
Field synergy analysis of pollutant dispersion in street canyons and its optimization by adding wind catchers
}

Tingzhen Ming ${ }^{1,2,3^{*}}$, Huina $\mathrm{Han}^{1}$, Zhen Zhao ${ }^{1}$, Renaud de Richter ${ }^{4}$, Yongjia $\mathrm{Wu}^{1}$, Wei $\mathrm{Li}^{5}$, Nyuk Hien Wong ${ }^{3}$

1. School of Civil Engineering and Architecture, Wuhan University of Technology, No.122 Luoshi Road, Hongshan District Wuhan 430070 China.

2. School of Architectural Engineering, Huanggang Normal University, No. 146 Xingang Second Road, Huanggang 438000 China.

3. School of design and environment, National University of Singapore, 4 Architecture Drive, 117566 Singapore sdewnh@nus.edu.sg

4. Tour-Solaire.Fr, 8 Impasse des Papillons, F34090 Montpellier, France

5. School of Engineering \& Applied Science, Aston University, Birmingham B4 7ET, United Kingdom

Corresponding authors:

Tingzhen Ming: Professor, School of Civil Engineering and Architecture, Wuhan University of Technology, No.122 Luoshi Road, Hongshan District Wuhan 430070 China. Email: tzming@whut.edu.cn

\begin{abstract}
The microenvironment, which involves pollutant dispersion of the urban street canyon, is critical to the health of pedestrians and residents. The objectives of this work are twofold: i) to effectively assess the pollutant dispersion process based on a theory and ii) to adopt an appropirate stratigy, i.e., wind catcher, to alleviate the pollution in the street canyons. Pollutant dispersion in street canyons is essentially a convective mass transfer process. Because the convective heat transfer process and the mass transfer process are physically similar and the applicability of field synergy theory to turbulence has been verified in the literature, we apply the field synergy theory to the study of pollutant dispersion in street canyons. In this paper, a computational fluid dynamics (CFD) simulation is conducted to investigate the effects of wind catcher, wind speed and the geometry of the street canyons on pollutant dispersion. According to the field synergy theory, Sherwood number and field synergy number are used to quantitatively evaluate the wind catcher and wind speed on the diffusion of pollutants in asymmetric street canyons. The results show that adding wind catchers can significantly improve the air quality of the step-down street canyon and reduce the average pollutant concentrations in the street canyon by $75 \%$. Higher wind speedenhances diffusion of pollutants differently in different geometric street canyons.
\end{abstract}

Keywords: Street canyon; Field synergy theory; CFD simulation; Pollutant dispersion; Wind catcher 


\section{Introduction}

As a result of rapid urbanization, the demand for transportation is increasing, resulting in increases in the emissions of motor vehicle pollutants and environmental pollution of urban street canyons. For an investigation of the dispersion characteristics of pollutants in street canyons, it is important to predict and evaluate air quality accurately based on urban morphology and meteorological conditions. Numerous computational fluid dynamics (CFD) simulations ${ }^{[1-5]}$, water/wind tunnel experiments ${ }^{[6,7]}$ and field measurements have been conducted to understand the impacts of various urban designs on urban airflow and pollutant dispersion.

A wide range of factors which have impact on urban pollutant dispersion have been studied, including building geometry ${ }^{[8,9]}$, local atmospheric conditions ${ }^{[10,11]}$, geometry of street canyons ${ }^{[12,}$ ${ }^{13]}$, chemical reactions of pollutants ${ }^{[14,15]}$, elevated building design ${ }^{[16,17]}$, thermal stratifications and buoyancy forces induced by wall heating and solar shading ${ }^{[18,19]}$ and tree plantings ${ }^{[20,21]}$ Specifically, for symmetric street canyons, the avenue street canyon coupled with short street canyon is beneficial to the dispersion of pollutants. For asymmetric street canyon, step-up street canyon and street canyon with non-uniform building heights are better for the microenvironment. For wind conditions, high wind speed and oblique wind direction are favorable conditions. For chemical reactions, it strongly depends on the layout of street canyons, atmospheric conditions and the presence of other chemicals. For the impact of the viaduct, the height of the viaduct should not be close to the depth of the street canyon. For thermal conditions, when the thermal stratification is unstable or when the windward side is the main heating surface, the air quality in street canyon would be better. For trees planting, planting low foliated trees and align discontinuously along the canyon would be a good choice.

Apart from the above factors, wind catchers have attracted particular research interest because they canenhance the ventilation of street canyon and improve the air quality in street canyons. As architectural elements, wind towers and wind catchers have historically been popular in the Middle East, especially Iran ${ }^{[22,23]}$. They are effective as passive cooling and natural ventilation methods for indoor/outdoor interfaces ${ }^{[24,25]}$, although air airflow rate is strongly influenced by geometry of wind catchers and wind direction and speed. Over the past few decades, some research has been carried out to revive and reuse wind catchers to improve thermal comfort ${ }^{[26,27]}$. In modern buildings, wind catchers are increasingly used as natural ventilation systems to minimize consumption of nonrenewable energy and reduce harmful emissions ${ }^{[28]}$. Montazeri et al. ${ }^{[29]}$ presented a detailed evaluation of the impact of outlet openings on basic flow characteristics of cross-ventilation using wind-catchers integrated into a single-zone isolated building. The results showed that using outlet openings very close to the wind catcher will not increase the induced airflow, while it leads to a considerable reduction in the indoor air quality. A combination of one-sided wind-catcher and window is superior, while the use of two-sided wind-catchers leads to the lowest indoor air quality and air change efficiency.

However, relevant research on the impact of wind catchers on pollutant dispersion in street canyon is still limited. Chew, et al. ${ }^{[30]}$ explored the potential of wind catchers to enhance air quality by installing a wind catcher prototype in a water channel. Experiments showed that the wind catchers enhance pedestrian-level wind speed in the target canyon by 2.5 times. Similarly, Zhang, et al. ${ }^{[31]}$ evaluated the effect of setting wind catchers on the decrease of vehicle pollution in deep street canyons through CFD simulation. The setting of the wind catchers greatly increased the wind 
speed in the upper area of the street canyon. In addition, the normalized speed of the pedestrian layer increased significantly, thereby reducing the $\mathrm{CO}$ concentration by one to two orders of magnitude. Under different traffic tidal flow conditions, the effect of wind catchers on street canyon structures was investigated by Li, et al. ${ }^{[32]}$. The results showed that in the shallow street canyon or regular street canyon, wind catchers can significantly reduce the personal intake fraction (IF_p), in the step-up or step-down street canyon, the decrease in windward IF_p was relatively slight with wind catchers.

The previous studies focused on the impact of the wind catchers on pedestrian-level, or on the windward or leeward side, this work intends to explore its impact on the overall pollutant diffusion in the target street canyon. More importantly, the above research tends to qualitatively analyze the impact of various physical factors on the diffusion of pollutants in the street canyon. However, the concern about the degree of influence of various factors on the diffusion of pollutants is not enough. We aim to address this gap and find that the convective mass transfer field synergy can be used to quantitatively evaluate the influence of different factors on the diffusion of pollutants in street canyon.

The theory of convection mass transfer field synergy comes from the theory of convection heat transfer field synergy. Therefore, the origin and development of this theory are summarized as follows. The global energy crisis that began in the early 1970s has led to the rapid development of heat transfer enhancement technology, but the resistance loss is also substantial, which limits the engineering application of this technology. Guo et al. ${ }^{[33]}$ compared a convective heat transfer system to a heat conduction system with an internal heat source in 1998 by integrating the energy equation of the boundary layer and introducing the concept of source strengthening. The theory of thermal field synergy resulted in new theories and new methods to enhance heat transfer. Han et al. ${ }^{\text {[34] }}$ demonstrated the thermodynamic, phenomenological, and philosophical basis of the field synergy theory based on the quiescent thermodynamic and non-equilibrium thermodynamic theory, and extended the field synergy theory to the three transfer processes in nature (momentum, heat, and mass transfer). The generalized field synergy theory was proposed. Liu et al. ${ }^{[35]}$ investigated the laminar heat transfer between a fluid and a wall surface and proposed the principle of synergy between the physical quantities of the particles in the flow field in laminar flow. The authors discussed the internal relationship between synergy and enhanced heat transfer and determined that any particle in the flow field can be characterized by the angle between the temperature gradient, velocity gradient, pressure gradient, and velocity vector, thereby providing theoretical guidance for the enhancement of convective heat transfer. Zeng and Tao ${ }^{[36]}$ applied the field synergy principle to turbulent heat transfer and calculated the field integration value to prove that the field synergy principle was also applicable to turbulent heat transfer. Liu et al. ${ }^{[37]}$ established the energy and momentum synergy equations of turbulent heat transfer based on the synergistic principle of laminar convective heat transfer and revealed the synergistic relationship between heat flow, mass flow, and the fluid driving force in turbulent heat transfer. The applicability of the field synergy principle to turbulent heat transfer was demonstrated. Chen et al. ${ }^{[38]}$ extended the field synergy principle to convection mass transfer analysis based on the analogy of the three transfer processes (momentum, heat, mass); the effect of the synergy between the velocity vector and the pollutant concentration gradient on the decontamination rate of indoor ventilation was demonstrated. The convective mass transfer field synergy equation was solved to obtain the best ventilation method, improve the room's purification capacity, and provide theoretical guidance for using the field synergy principle to 
improve the decontamination rate.

In summary, we apply convection mass field synergy theory to quantitatively analyze pollutant diffusion, i.e. to measure the effect of wind catchers, wind speed, step-up and step-down street canyons on pollutant diffusion. Right-angle wind catchers were added to buildings on both sides of street canyons with different geometric shapes to improve ventilation capacity of street canyons, Field synergy angle and Sherwood number are used to measure the effect of wind catchers on pollutant dispersion in street canyons. Field synergy number and Sherwood number are used to evaluate the effect of geometry of street canyon and wind speed on pollutant dispersion. It is worth noting that we performed two experimental verifications in order to increase reliability of numerical simulation results...

\section{Field synergy theory in convective mass transfer}

Chen et al. ${ }^{[38]}$ used the concentration conservation equation of the steady-state mass diffusion of the three-dimensional passive term and integrated the equation over the entire diffusion domain. Gauss's theorem was then used to convert the volume integral to the surface integral. On a surface without mass diffusion, the concentration gradient is zero, and the integral value is zero; the inlet and outlet wind speeds are very large, but the concentration gradient is small and can be ignored. The final equation is simplified as:

$$
\int_{\Omega} \rho|U||\nabla C| \cos \beta d V=\int_{S} \vec{n}(\rho \mathrm{D} \nabla \mathrm{C}) d S
$$

where $\rho$ represents the air density $\left(\mathrm{kg} / \mathrm{m}^{3}\right) ; U$ represents the fluid velocity $(\mathrm{m} / \mathrm{s}) ; \nabla \mathrm{C}$

represents the concentration gradient of the component; $D$ represents the mass transfer diffusion

coefficient $\left(\mathrm{m}^{2} / \mathrm{s}\right) ; \quad \beta$ represents the angle between the velocity vector and the vector of the concentration gradient of the component.

The dimensionless velocity and concentration gradient are defined as:

$$
\begin{aligned}
& \bar{U}=\frac{\vec{U}}{\sqrt{U_{x}^{2}+U_{y}^{2}}} \\
& \overline{\nabla C}=\frac{\overline{\nabla C}}{\sqrt{\nabla C_{x}^{2}+\nabla C_{y}^{2}}}
\end{aligned}
$$

The dimensionless variables are substituted into equation (1). The equation is dimensionless and the Sherwood number, which represents the relative size of convection and diffusion mass transfer, is obtained. The pollutant dispersion in street canyons is influenced by various factors such as ambient wind, non-isothermal effect caused by solar radiation, vehicle movement and its high temperature exhaust emissions, geometrical configurations of the street canyons. Under the combined influences of the above factors, pollutants are mainly spread in the form of convection with the air in the street canyon, which is a typical mass transfer process. The equation of the Sherwood number is as follows: 


$$
S h=\operatorname{Re} S c \frac{\int_{\Omega} \bar{U} \cdot \overline{\nabla C} d V}{V}
$$

The Sherwood number is $S h=\frac{h_{m} d}{D}$ and the Schmitt number is $S c=\frac{v}{D}$; these numbers are related to the physical properties of fluid $; h_{m}$ represents the convective mass transfer coefficient.

Equation (4) indicates that the Sherwood number is not only related to the Reynolds number and Schmitt number but also depends on the synergy angle between the velocity vector and the concentration gradient vector, which represents the integral value of the dot product of the two vectors in the target area. Therefore, the integral value, which represents the synergism, is defined as the field synergy number.

$$
F \mathrm{c}_{m}=\frac{\int_{\Omega} \bar{U} \cdot \overline{\nabla C} d V}{V}
$$

In equation (5), $\bar{U} \cdot \overline{\nabla C}=|\bar{U}||\overline{\nabla C}| \cos \theta$. The larger the dot product of the two vectors, the larger the field synergy number, the larger the Sherwood number, and the better the convective mass transfer effect are. The expression of the field synergy angle is derived as follows:

$$
\theta=\arccos \left(\frac{\bar{U} \cdot \overline{\nabla C}}{|\bar{U}||\overline{\nabla C}|}\right)
$$

\section{Physical model and mathematical model}

\subsection{Physical model}

An urban multi-street canyon model was established (Fig. 1) to analyze the influence of different street canyon shapes on pollutant dispersion. There are seven street canyons in the model, and the fourth street canyon is the target street canyon. The different street canyon shapes are set only for the target street canyon, and other street canyons remain unchanged. The building height is $\mathrm{H}=20 \mathrm{~m}$, and the ideal street canyon model $\mathrm{H} / \mathrm{W}=1: 1$ is used. A two-way, four-lane street is located in the center of the canyon. Each lane has a width of $4 \mathrm{~m}$ and a total length of $16 \mathrm{~m}$. A uniform surface pollution source is assumed in the lane, with a width of $0.8 \mathrm{H}$ and a height of 0.03 $\mathrm{H}$. The inlet boundary is $5 \mathrm{H}$ from the upstream building of the first street canyon, the outlet boundary is $10 \mathrm{H}$ from the downstream building of the 7th street canyon, and the upper boundary is $5 \mathrm{H}$ from the top of the building. Based on the above criteria, the size of the computational domain is $520 \mathrm{~m} \times 120 \mathrm{~m}$. 


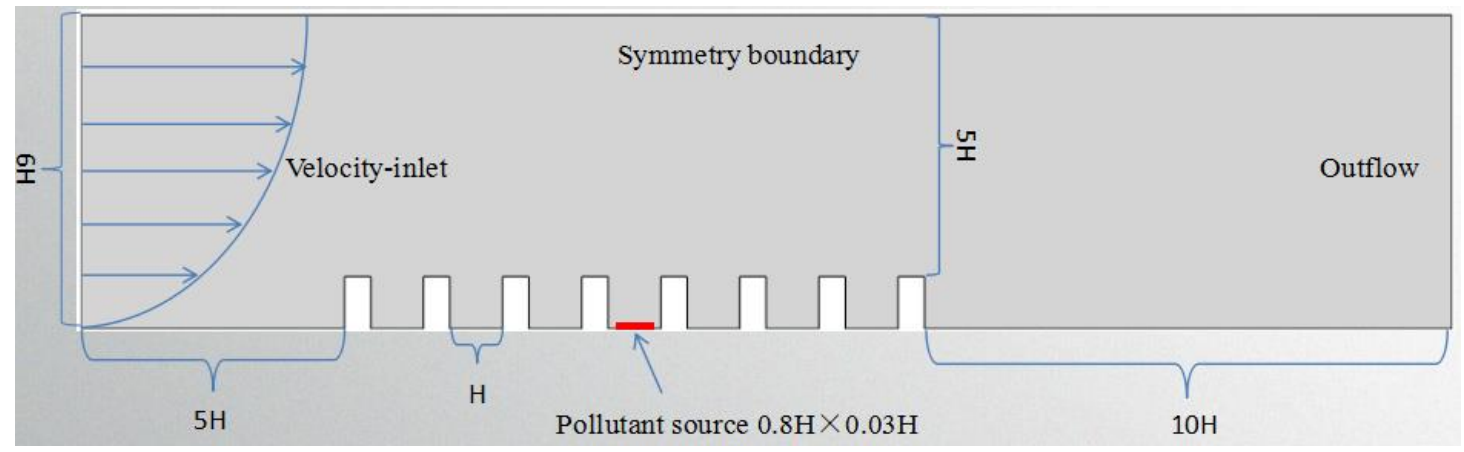

Fig. 1 Computing domain and boundary conditions of the street canyon model

\subsection{Mathematical model}

At present, numerical simulations of street canyons have commonly used the Reynoldsaveraged Navier-Stokes turbulence model. Scholars ${ }^{[39,40]}$ have compared the characteristics of different Reynolds-averaged turbulence models. The renormalized $\mathrm{k}-\varepsilon$ model is a modification of the standard k- $\varepsilon$ model, and it was found that the former model provides more accurate results for the prediction of pollutant concentration. Therefore, we use the renormalized k- $\varepsilon$ turbulence model to simulate pollutant dispersion.

In street canyons, the air moves at a slower speed than elsewhere, and the air is considered to be an incompressible fluid. The flow of viscous incompressible fluids is commonly described by the Navier-Stokes equation. It is assumed that the incoming wind and source intensities do not change over time. The continuity equation, momentum equation, energy equation, pollutant transport equation, and the renormalization group (RNG) $k-\varepsilon$ equation are defined as follows:

Continuity equation:

$$
\frac{\partial u_{i}}{\partial x_{i}}=0
$$

Navier-Stokes equation:

$$
\begin{aligned}
& \frac{\partial}{\partial t}\left(\rho u_{i}\right)+\frac{\partial}{\partial x_{j}}\left(\rho u_{i} u_{j}\right)=-\frac{\partial p}{\partial x_{i}}+\frac{\partial \tau_{i j}}{\partial x_{j}}+\rho g_{i}+F_{i} \\
& \tau_{i j}=\left[\mu\left(\frac{\partial u_{i}}{\partial x_{j}}+\frac{\partial u_{j}}{\partial x_{i}}\right)\right]-\frac{2}{3} \mu \frac{\partial u_{i}}{\partial x_{i}} \delta_{i j}
\end{aligned}
$$

Energy equation:

$$
\frac{\partial}{\partial t}(\rho E)+\nabla \bullet(\vec{v}(\rho E+p))=\nabla \bullet\left(\mathrm{k}_{e f f} \nabla \mathrm{T}-\sum h_{j} \bar{J}_{j}+\left(\overrightarrow{\bar{\tau}}_{\text {eff }} \bullet \vec{v}\right)\right)+S_{h}
$$

where $k_{\text {eff }}$ represents the effective thermal conductivity. $\bar{J}_{j}$ represents the component's diffusion flux. The first three terms on the right of equation (13) represent the energy transfer due to heat conduction, component diffusion, and viscous dissipation, respectively. $\mathrm{S}_{\mathrm{h}}$ denotes the chemical reaction's exothermic (endothermic) heat and any other user-defined volumetric heat source. 
Pollutant transport equation:

$\frac{\partial C_{i}}{\partial t}+u_{j} \frac{\partial C_{i}}{\partial x_{j}}=\frac{1}{\rho} \frac{\partial}{\partial x_{j}}\left[\left(\mathrm{D}_{i}+\frac{\mu_{t}}{S c_{t}}\right) \frac{\partial C_{i}}{\partial x_{j}}\right]+S$

$\mathrm{S}$ represents the pollutant source term defined in this study, which is $10^{-7} \mathrm{~kg} /\left(\mathrm{m}^{3} . \mathrm{s}\right)$.

RNG k- $\varepsilon$ equation:

$$
\begin{aligned}
& \frac{\partial}{\partial t}(\rho k)+\frac{\partial}{\partial x_{i}}\left(\rho k u_{i}\right)=\frac{\partial}{\partial x_{j}}\left(\alpha_{k} \mu_{e f f} \frac{\partial k}{\partial x_{j}}\right)+G_{k}+G_{b}-\rho \varepsilon-Y_{M}+S_{k} \\
& \frac{\partial}{\partial t}(\rho \varepsilon)+\frac{\partial}{\partial x_{i}}\left(\rho \varepsilon u_{i}\right)=\frac{\partial}{\partial x_{j}}\left(\alpha_{\varepsilon} \mu_{e f f} \frac{\partial \varepsilon}{\partial x_{j}}\right)+C_{1 \varepsilon} \frac{\varepsilon}{k}\left(G_{k}+C_{3 \varepsilon} G_{b}\right) \\
& -\left[\rho C_{2 \varepsilon}+\frac{C_{\mu} \rho \eta^{3}\left(1-\eta / \eta_{0}\right)}{1+\beta \eta^{3}}\right] \frac{\varepsilon^{2}}{k}+S_{\varepsilon}
\end{aligned}
$$

where $\alpha_{k}$ represents the inverse effective Prandtl number of $\mathrm{K}, \alpha_{\varepsilon}$ represents the inverse effective Prandtl number of $\varepsilon$, and $\mu_{e f f}$ represents the effective turbulent viscosity; $\eta=S k / \varepsilon, S$ represents the deformation tensor, $C_{1 \varepsilon}=1.42, C_{2 \varepsilon}=1.68, C_{\mu}=0.0845, \beta=0.012, \quad \eta_{0}=4.38$.

\subsection{Boundary conditions}

The velocity inlet is in the form of a gradient wind, and the outlet boundary is set to a free-flow condition. The upper boundary of the computational domain is set to a symmetric boundary condition, which is a zero-gradient boundary. In the near-wall area and near-ground area of the building, turbulence cannot be fully developed. On the wall, roof, and ground of the building, a standard wall function is introduced for near-wall correction. All the other wall surfaces shall be applied with a slip wall boundary condition.

The vertical characteristics of the ambient wind are affected by the terrain and are closely related to the roughness of the terrain. Based on this, the gradient wind is used to represent the ambient wind speed. For the inlet boundary condition, the wind speed conforms to the law of exponents or the law of logarithms ${ }^{[41,42]}$. In this paper, the user-defined function is used to define the exponential rate velocity curve of the velocity inlet and the corresponding turbulent kinetic energy and turbulent dissipation rate as follows:

$$
\begin{aligned}
& U_{z}=U_{r e f}\left(\frac{z}{z_{r e f}}\right)^{\alpha} \\
& k=\frac{\left(\mathrm{U}^{*}\right)^{2}}{\sqrt{C_{\mu}}} \\
& \varepsilon=\frac{\left(\mathrm{U}^{*}\right)^{3}}{k z}
\end{aligned}
$$

where $U_{r e f}$ represents the velocity at a reference height. $\mathrm{U}^{*}$ represents the friction velocity. $k$ 
represents the von Karman constant (0.4), and $\mathrm{C} \mu=0.09$.

\subsection{Mesh generation and sensitivity analysis}

The discretization of the computing domain is an important aspect of numerical simulations. The advantage of a structured mesh ${ }^{[43]}$ is that the mesh is regular, simple, and convenient, the calculation is easy, and the memory requirement is small. Because the model in this study is relatively simple and regular, structured meshes were used as the computing domain to ensure the accuracy of the calculation and minimize the memory requirements. Eight-layer boundary meshes are used on the wall surface and the ground of the building to ensure the applicability of the standard wall surface function. The first layer mesh is $0.02 \mathrm{~m}$, and the $\mathrm{y}+$ at the boundary is guaranteed to be about 20. Inside the street canyon, the mesh division of the boundary area of the computing domain is relatively sparse; the mesh of $\Delta \mathrm{x} \times \Delta \mathrm{y}=0.2 \mathrm{~m} \times 0.2 \mathrm{~m}$ is used. The final mesh is shown in Fig. 2 .

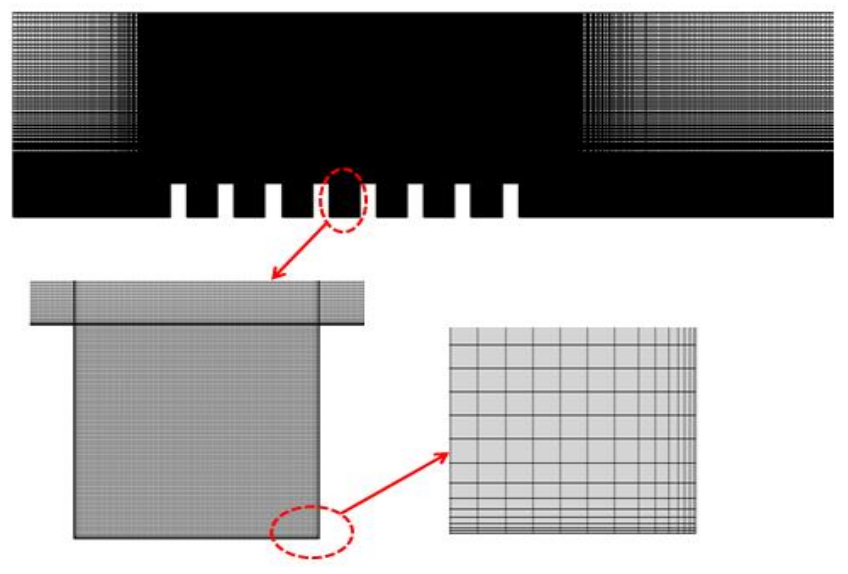

Fig. 2 Schematic diagram of the mesh demarcation

The commercial CFD software ANSYS FLUENT 15.0 is used for the numerical simulation. The solution uses the SIMPLE algorithm to compile the ambient wind through the user-defined function. The second-order upwind style is used for the discrete method of the convection term, the Green-Gaussian node method is used for gradient interpolation, and the standard algorithm is used for pressure interpolation. The convergence factor of the energy equation is $1 \times 10^{-8}$, and the convergence factors of the other variables are $1 \times 10^{-5}$.

The independence of the mesh is validated to ensure accurate results. Three different mesh sizes $(201162,272608$, and 396578) are selected. The calculations are performed on the same computer under the same working conditions. The reference wind speed is $2 \mathrm{~m} / \mathrm{s}$, and the source intensity of the pollution source is $10^{-7} \mathrm{~kg} /\left(\mathrm{m}^{3} \cdot \mathrm{s}\right)$. We select the centerline of the street in the X-axis direction $(X=210 \mathrm{~m}, \mathrm{Y}$ line from $0 \mathrm{~m}-20 \mathrm{~m})$. The wind velocity and $\mathrm{CO}$ mass fraction for different mesh sizes are shown in Figure 3 (a) and (b), respectively. The maximum errors between the different mesh sizes for the wind velocity and CO mass fraction are less than 5\%. The results show that a further increase in the mesh size will not provide a significant change in the calculation results. Therefore, the mesh size of 272608 is selected in this study. 


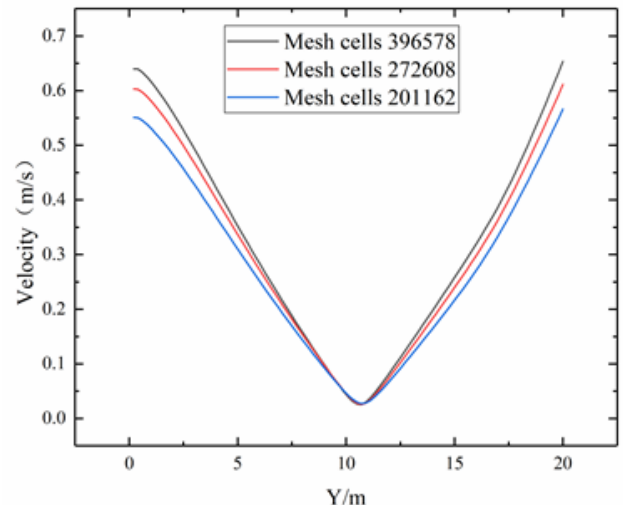

(a)

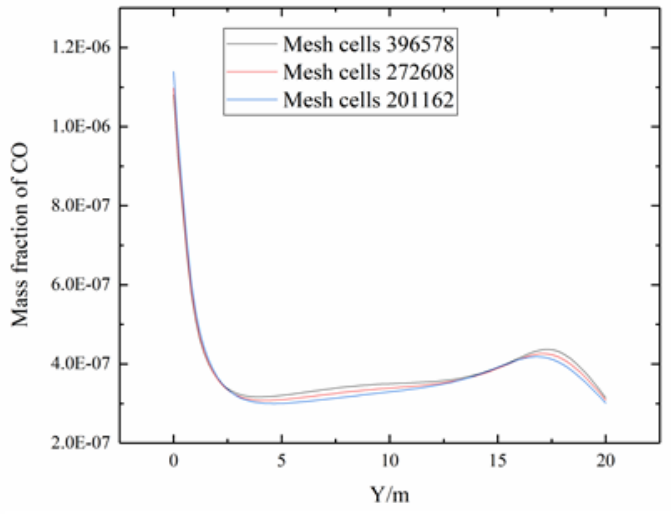

(b)

Fig. 3 (a) wind velocity and (b) mass fraction of $\mathrm{CO}$ on the $\mathrm{X}=210 \mathrm{~m}$ line for different mesh sizes

\subsection{Model validation}

Model validation is an important component of simulations to ensure the accuracy and reliability of the numerical simulation results. The most common method is to establish a model that has the same parameters as those of a wind tunnel test, use the same boundary conditions and working conditions, and compare the results. In order to increase reliability, two experimental verifications were performed. The first experiment verifies the applicability of the geometric model through wind speed profiles. The second verifies the reliability of simulated pollutant concentrations.

In the first validation, we use the wind tunnel experimental data of Takenobu et al. ${ }^{[44]}$ to evaluate the reliability of the two-dimensional multi-street canyon application of the RNG k- $\varepsilon$ model. The wind tunnel experiment was conducted at the Japanese Central Electric Power Research Institute. The size of the experimental wind tunnel was $17 \mathrm{~m} \times 3 \mathrm{~m} \times 1.7 \mathrm{~m}$. At the test entrance (h $=0.65 \mathrm{~m}$ ), 7 Irwin-type vortex generators were located, and $3 \mathrm{~L}$-shaped partitions were arranged at equal intervals on the ground from $\mathrm{X}=0$ to $\mathrm{X}=5.0 \mathrm{~m}$ at the entrance. Twenty-eight identical (1.56 $\mathrm{m} \times 0.12 \mathrm{~m} \times 0.12 \mathrm{~m}$ ) cubes with equal spacing of $0.12 \mathrm{~m}(\mathrm{H})$ were located at a distance of $10.5 \mathrm{~m}$ from the air outlet to simulate multiple street canyons and produce near-surface turbulent flow. A gradient wind was set at the inlet boundary, and the reference wind speed at $\mathrm{z}=2 \mathrm{H}$ was $1.15 \mathrm{~m} / \mathrm{s}$; the 25th street canyon was used as the test street canyon, and the change in wind speed in the Z-axis direction at $\mathrm{Y} / \mathrm{H}=0, \mathrm{X} / \mathrm{H}=0.25,0.5,0.75$ of the tested street canyon was measured using a laser Doppler velocimeter.

In the second validation, the concentrations obtained by RANS is compared with those in the experiments conducted by Pavageau and Schatzmann ${ }^{[45]}$. The pollutant emission rate $\mathrm{Q}$ is represented by a ground-level continuous pollutant line source of length Ly placed parallel to the spanwise axis from $\mathrm{X} / \mathrm{H}=0.0$ to $\mathrm{X} / \mathrm{H}=1.0$. The tracer gas ethane (which is used in this wind-tunnel experiment and also our numerical simulation) is only emitted from a line source within the canyon. As shown in $\mathrm{x}$ axis of Figure 5, concentration $\mathrm{K}$ is normalized by the $\mathrm{U}_{\text {ref }}$ (the free-stream velocity, $1 \mathrm{~m} / \mathrm{s}$ ) taken at $650 \mathrm{~mm}$ above the floor in the free-stream region of the flow above the test street. C is the actual measured concentration, block height $\mathrm{H}(0.06 \mathrm{~m})$, line source length $\mathrm{L}$, and total emission Qe.

Based on the two experiments, the same geometric models were established in this study, and consistent boundary conditions were set. The calculation results are shown in Figure 4 and Figure 5. For the fluid velocity in Figure 4, the simulation data agree well with the experimental data. 
However, at $\mathrm{z} / \mathrm{H}<0.4$, the simulated values are slightly higher than the experimental values. As to the concentration, Figure 5 shows that the numerical simulation results of normalized mean concentration at $\mathrm{X} / \mathrm{H}=0.0$ agree well with the experiment data. However, there is a small deviation in the normalized mean concentration at $\mathrm{X} / \mathrm{H}=1.0$, which may be due to the difference in the airflow generated by the vortex generator and the simulated airflow. Despite some slight differences, the RNG k- $\varepsilon$ turbulence model is feasible for solving fluid flow and pollutant dispersion.

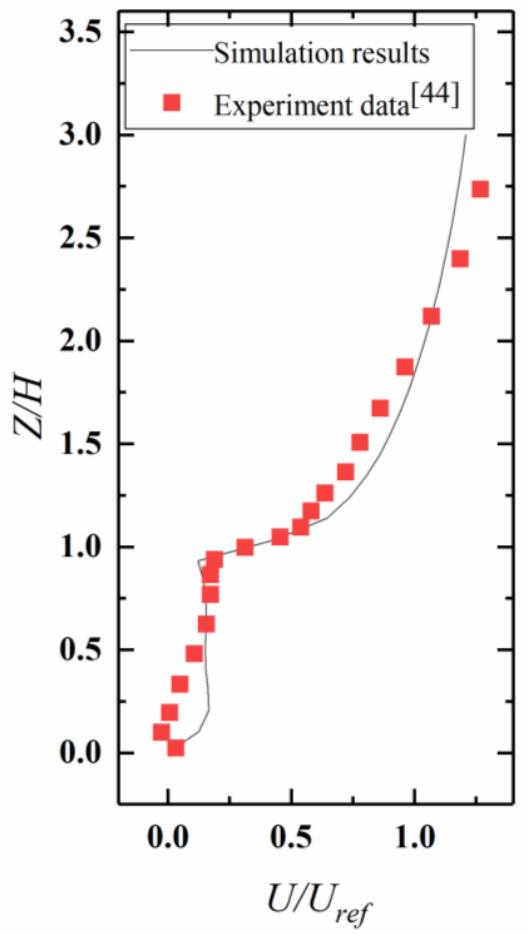

(a) $\mathrm{x} / \mathrm{H}=0.25$

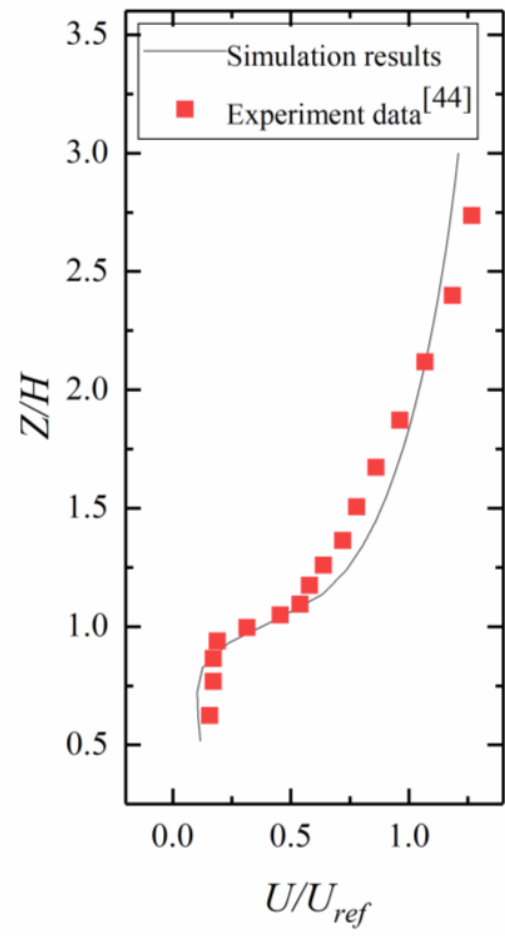

(b) $\mathrm{x} / \mathrm{H}=0.75$

Fig. 4 Comparison of the normalized velocity in the vertical direction obtained from simulations and experiments ${ }^{[44]}$. 


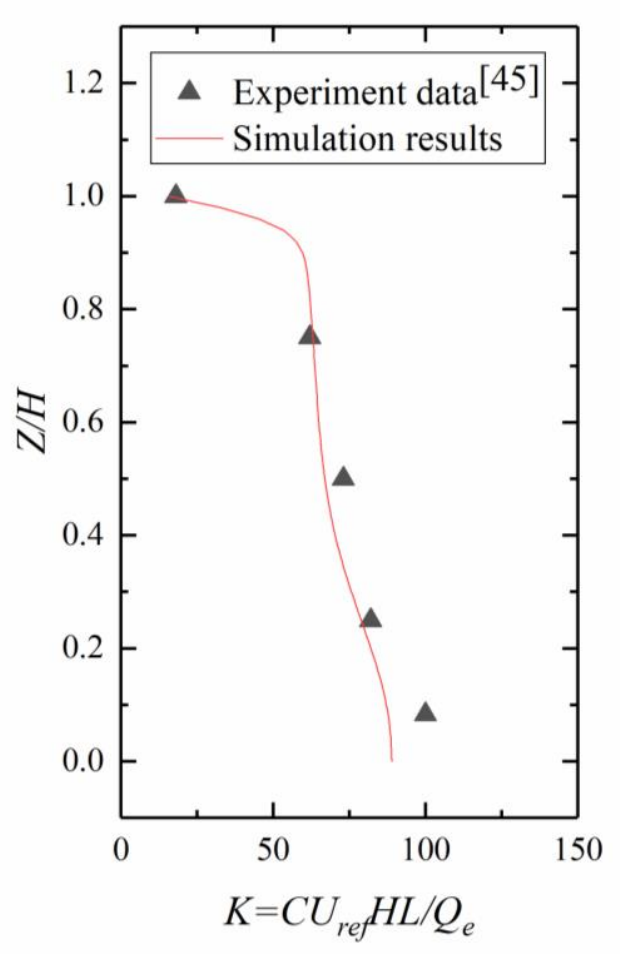

(a) $\mathrm{x} / \mathrm{H}=0.0$

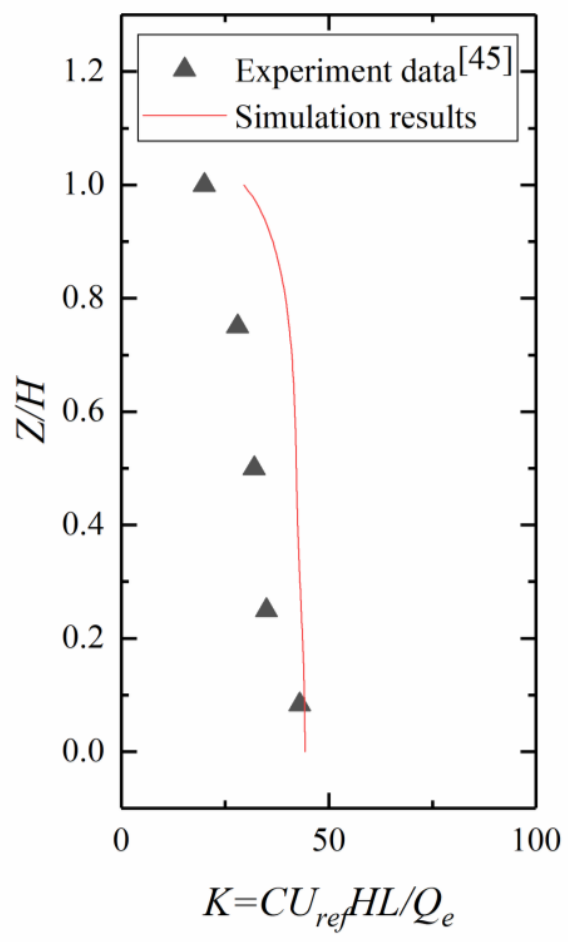

(b) $\mathrm{x} / \mathrm{H}=1.0$

Fig. 5 Comparison of the normalized concentration in the vertical direction obtained from simulations and experiments ${ }^{[45]}$.

\section{Results and discussion}

Pollutants in street canyons can be divided into gaseous pollutants and solid particles. Compared with gaseous pollutant, the dispersion effect of particulate matter is not only affected by wind speed and thermal buoyancy but is also closely related to the particle size of the particulate matter and the deposition effect, which depends on the air humidity. For simplification, CO is selected as the pollution source because it accounts for a relatively large part of the vehicle's exhaust gases and has stable chemical properties. For comparison and analysis, the concentration of the pollutant is dimensionless and is defined as follows:

$K=\frac{\rho_{a i r} C U_{r e f} H}{S_{c} A_{c}}$

where $\mathrm{C}$ represents the mass fraction of the pollutants obtained from the FLUENT simulation; $U_{r e f}(\mathrm{~m} / \mathrm{s})$ is the wind speed at the reference height; $H$ represents the building height, $S_{c}$ represents the total emission intensity of the pollutant source $\left(\mathrm{kg} /\left(\mathrm{m}^{3} . \mathrm{s}\right)\right), A_{c}\left(\mathrm{~m}^{2}\right)$ represents the area of the pollutant source. The concentration of the pollutants is dimensionless to avoid the influence of the source intensity and street geometry on the calculation results.

The equation for calculating the average pollutant concentration in the street canyon is:

$$
\bar{C}=\frac{\int_{\Omega} C_{i} d S}{S_{\Omega}}
$$


where $C_{i}$ represents the average mass fraction of $\mathrm{CO}$ in the i-th grid; $\Omega$ represents the computing domain of the target street canyon; $S_{\Omega}$ represents the area of the target street canyon.

\subsection{Influence of street canyon geometry}

The building heights are different on different sides of the street in real life. In this study, we analyzed the pollutant dispersion in three common street canyon types, namely the step-up street canyon $(\mathrm{H} 1: \mathrm{H} 2=1: 2)$, the symmetrical street canyon $(\mathrm{H} 1: \mathrm{H} 2=1: 1)$, and the step-down street canyon $(\mathrm{H} 1: \mathrm{H} 2=2: 1)$. The field synergy theory is used for the quantitative analysis of the relationship between pollutant dispersion and convective mass transfer in street canyons.

Figure 6 shows that the change in the street canyon shape caused significant changes in the flow field and the distribution of pollutants. The following was observed: (1) there is a clockwise main vortex in the step-up street canyon (fig6(a) and fig6(b)). Due to the low buildings on the leeward side, the air flows out of the street canyon toward the leeward building and interacts with the air in the adjacent street canyon on the left, forming a small vortex on the leeward building roof. The pollutant is concentrated near the ground on the leeward side, near the building surface, and on the roof. Near the building on the windward side, including the right sidewalk, the concentration of pollutants is consistent with the background concentration. (2) There is a closed clockwise main vortex inside the symmetrical street canyon (fig6(c) and fig6(d)). The concentration of pollutants on the leeward side is greater than that on the windward side. The impact of the pollutants on the leeward building is limited to the street-facing façade without affecting the roof. The concentration of pollutants near the windward building and the ground is slightly higher than the background concentration. (3) There are two vortices with opposite directions in the step-down street canyon (fig6(e) and fig6(f)). The pollutant is concentrated near the windward building. Due to the suppression of the upper vortex, most of the pollutants are concentrated in the lower right of the street canyon. The concentration near the leeward building is stratified, and the concentration of pollutants in the lower half is nearly twice that in the upper half. By comparing the maximum dimensionless concentration of the three types of street canyons, the step-up street canyon has the lowest pollutant concentrations, and the step-down street canyon has a concentration that is about twice that of the other two street canyon types. The results show that the step-up street canyon contributes to the dispersion of pollutants, whereas the step-down street canyon hinders the dispersion of pollutants.

The dispersion of pollutants in the air is essentially a process of convective mass transfer. We introduce the concept of the field synergy theory of the convective mass transfer to determine the characteristics of pollutant dispersion in street canyons. The theory indicates that for the same substance and the same dispersion conditions, its physical properties are unchanged; therefore, the mass transfer dispersion coefficient and kinematic viscosity are constant. The effect of the convective mass transfer depends not only on the magnitude of the velocity vector and the concentration gradient vector but also on the angle of the two vectors. For the three types of street canyons, the reference wind speed $(2 \mathrm{~m} / \mathrm{s})$, the Reynolds number, and the Schmitt number are the same. The quantitative parameter that characterizes the effect of the convective mass transfer is the field synergy number $\mathrm{F}_{\mathrm{cm}}$. 


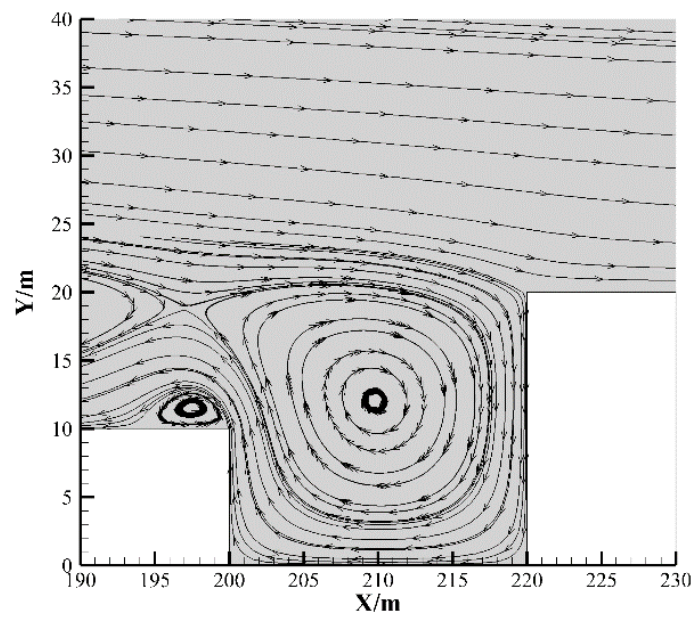

(a)

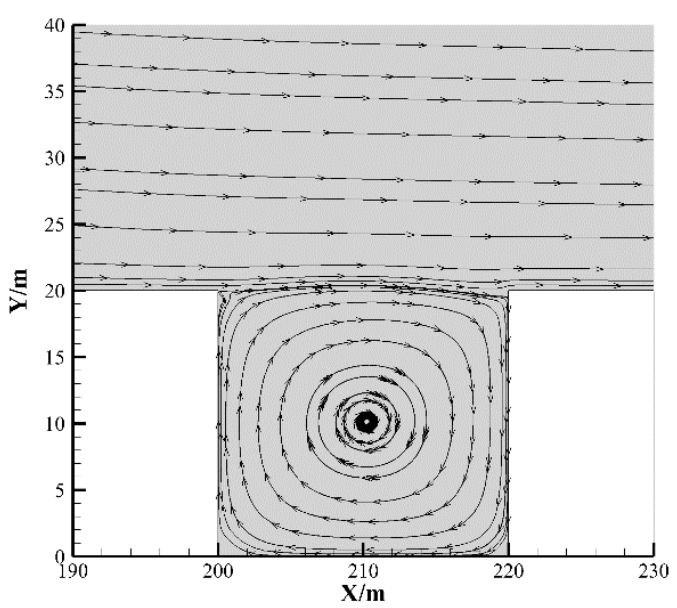

(c)

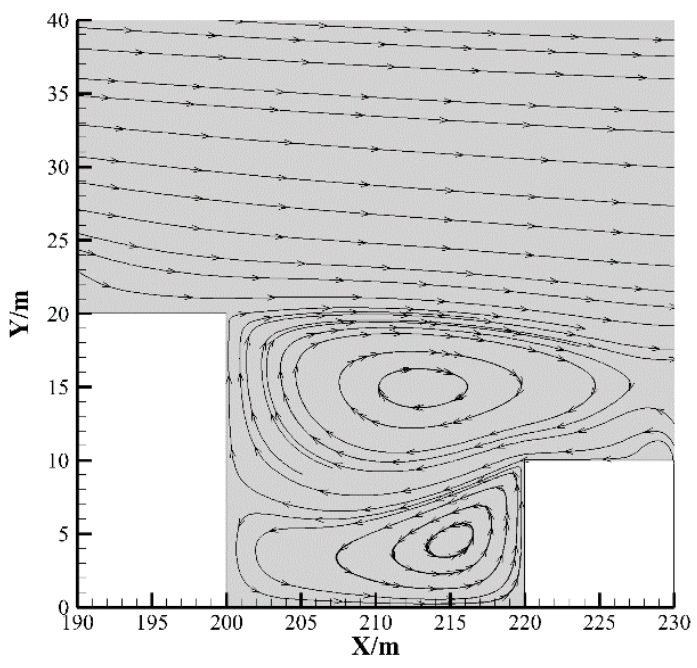

(e)

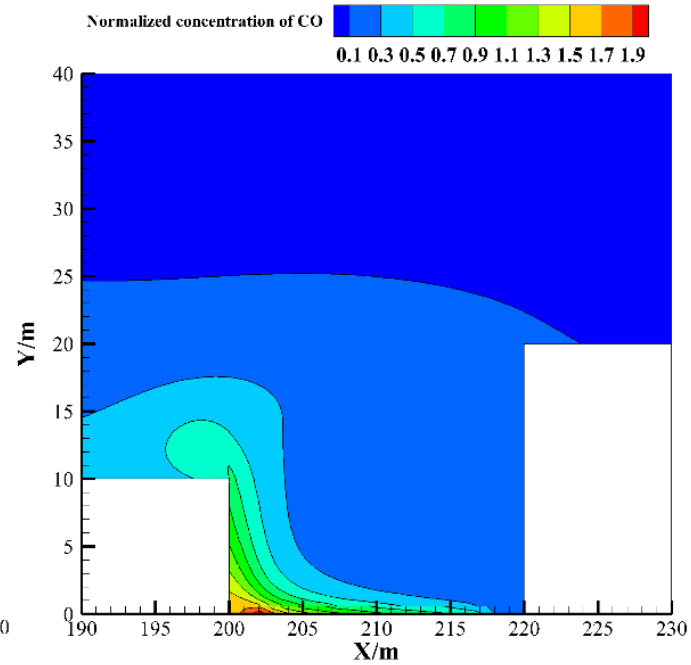

(b)

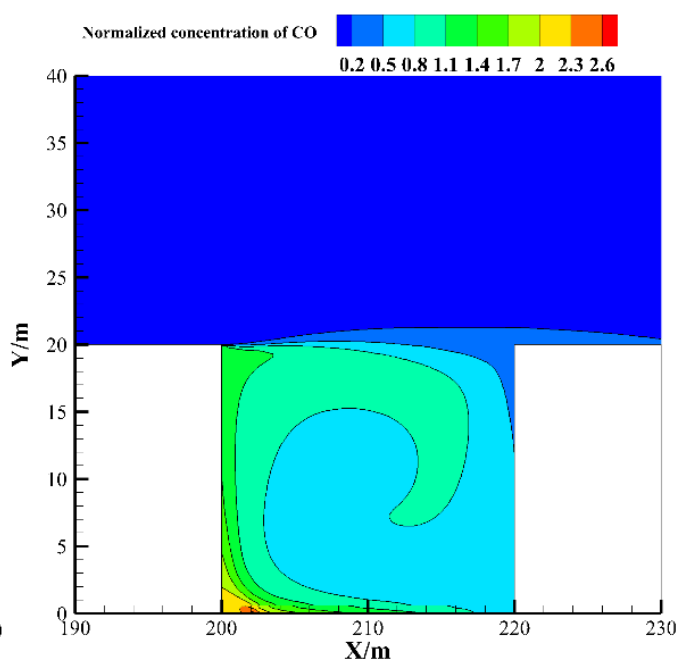

(d)

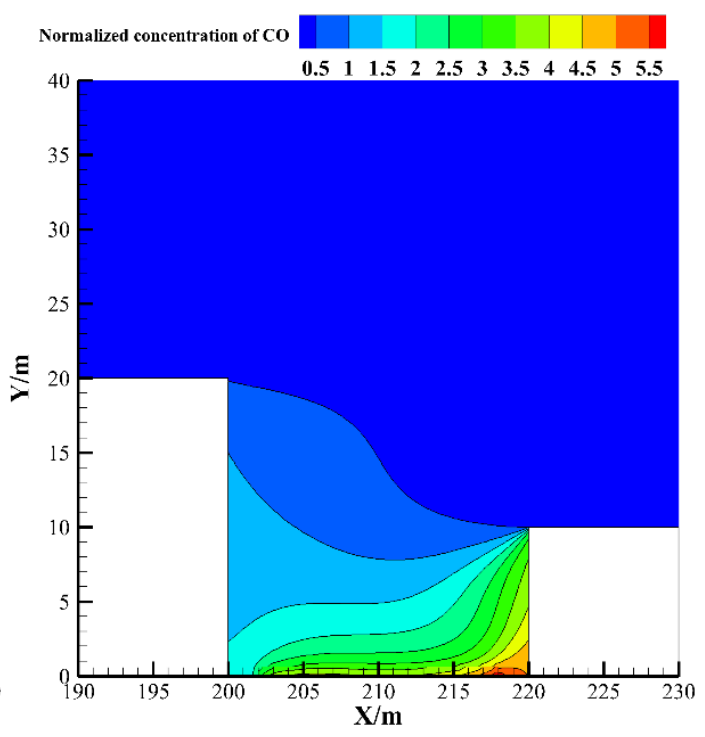

(f)

Fig. 6 Flow fields and dimensionless concentrations of CO in three street canyons.(a)and(b) step- 
up street canyon; (c)and(d), symmetrical street canyon; (e)and(f,) step-down street canyon.

Figure 7 shows the histogram of the field synergy number and the average $\mathrm{CO}$ concentration in the three street canyons. The results show that the larger the field synergy number, the smaller the average $\mathrm{CO}$ concentration is in the street canyon. The reason is that the better the synergy between the velocity field and the concentration gradient field is in the street canyon, the better the convective mass dispersion effect is. The results show that the use of convection mass transfer theory is suitable for the quantitative analysis of the pollutant dispersion characteristics in street canyons with different geometries. The pollutant dispersion capacity in the three types of street canyons from best to worst is step-up street canyon $>$ symmetrical street canyon $>$ step-down street canyon.

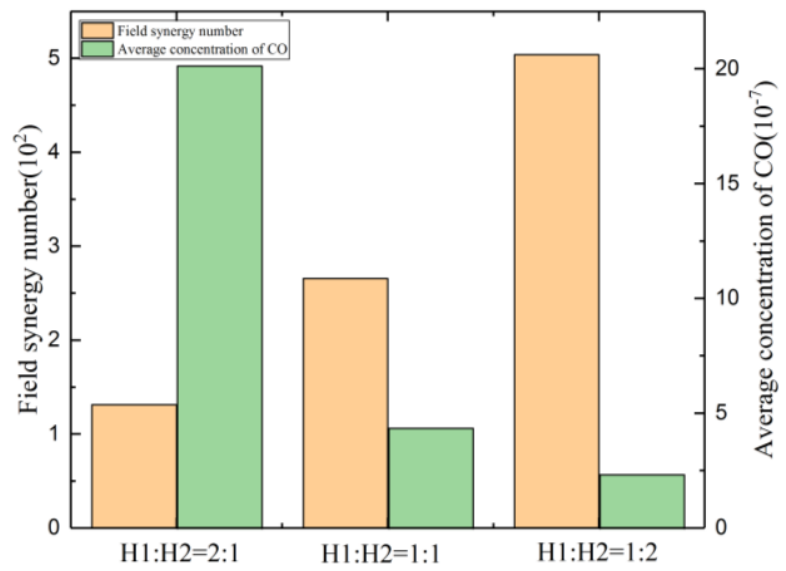

Fig. 7 Histogram of field synergy number and average concentration

\subsection{Influence of the wind catcher}

Air quality in street canyons is closely related to ventilation. In the Middle East and North Africa, wind towers and wind catchers were once common building elements because they are highly effective methods for natural ventilation and passive cooling. Researchers ${ }^{[31,46]}$ have studied the impact of wind catchers on urban ventilation and pollutant dispersion in urban street canyons. The geometry and wind direction of the wind catcher are the primary factors affecting the airflow in street canyons. We investigated the use of wind catchers $(0.3 \mathrm{H} \times 0.5 \mathrm{H})$ (Figure 8$)$ in the three street canyon types to determine the ventilation effect and the pollutant dispersion characteristics.

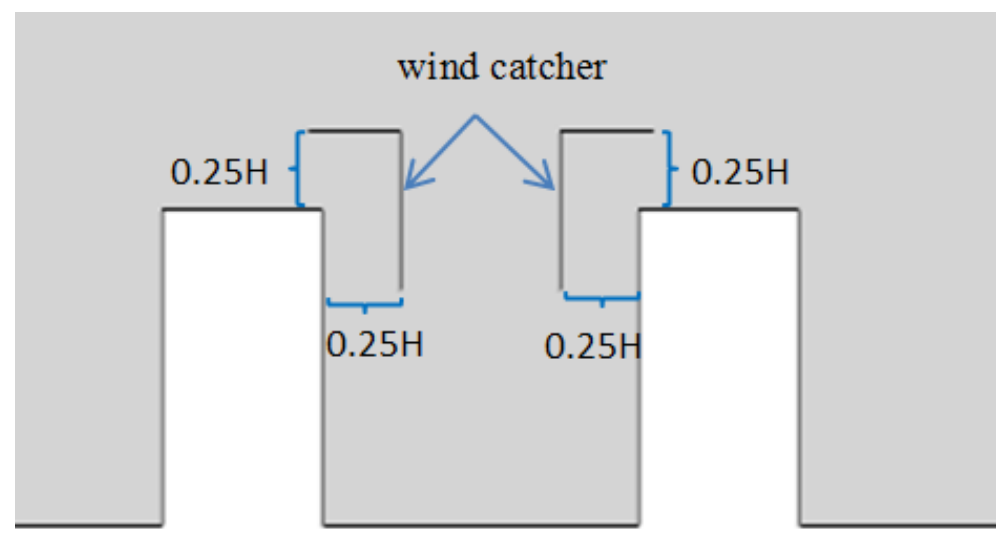

Fig. 8 Diagram of the wind catchers

Figure 9 shows that the setting of the wind catcher changes the flow field structure and vortex 
structure in the street canyon and forms a channel flow so that the air can flow out of the street canyon, which improves the ventilation in the street canyon. The following was observed: (1) In the step-up street canyon(fig9(a) and fig9(b)), the original airflow was divided into two parts; one part flowed out of the adjacent street canyon on the windward side, and the other flowed out of the adjacent street canyon on the leeward side. The concentration of pollutants on the leeward side of the street canyon was uniformly distributed in the near-surface area of the street canyon, which is detrimental to the health of pedestrians. (2) In the symmetrical street canyon (fig9(c) and fig9(d)), the distribution of the pollutants changed from the leeward side to the windward side, and some of the pollutants were moved out of the street canyon; this resulted in a decrease in the area affected by the pollutants. (3) In the step-down street canyon(fig9(e) and fig9(f)), the original counterclockwise vortex in the lower part of the street canyon changed into a channel flow, and the pollutants originally concentrated near the windward side of the street canyon dispersed out of the street canyon. As shown in Fig. 9(f), most of the pollutants were located near the wind catcher on the right, and the $\mathrm{CO}$ concentration was reduced from the original 5.5 to 3.2 , indicating an improvement in the air quality in the step-down street canyon.

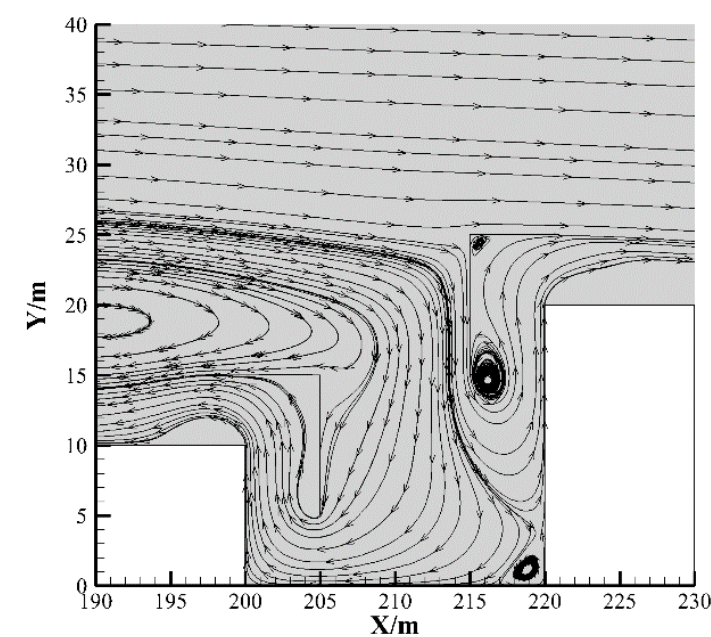

(a)

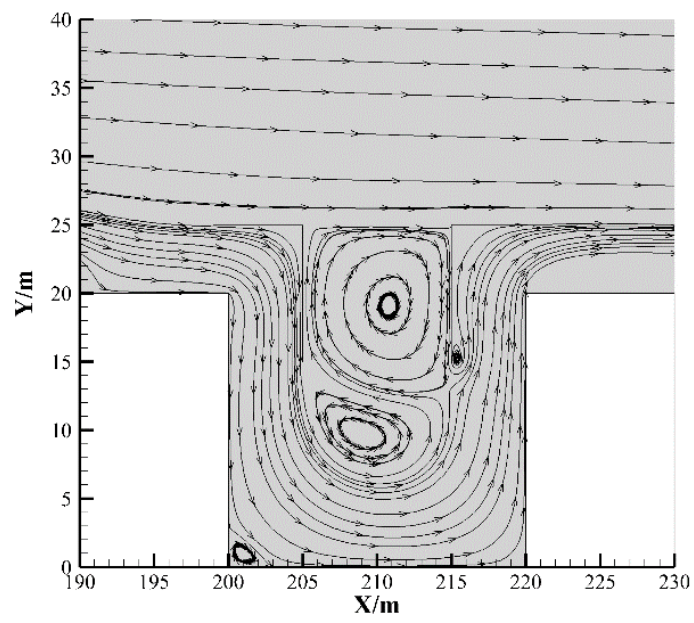

(c)

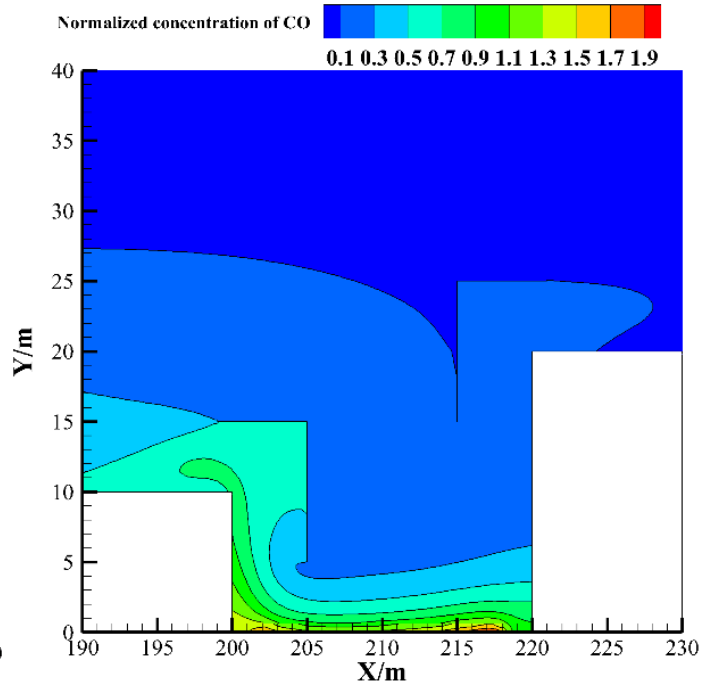

(b)

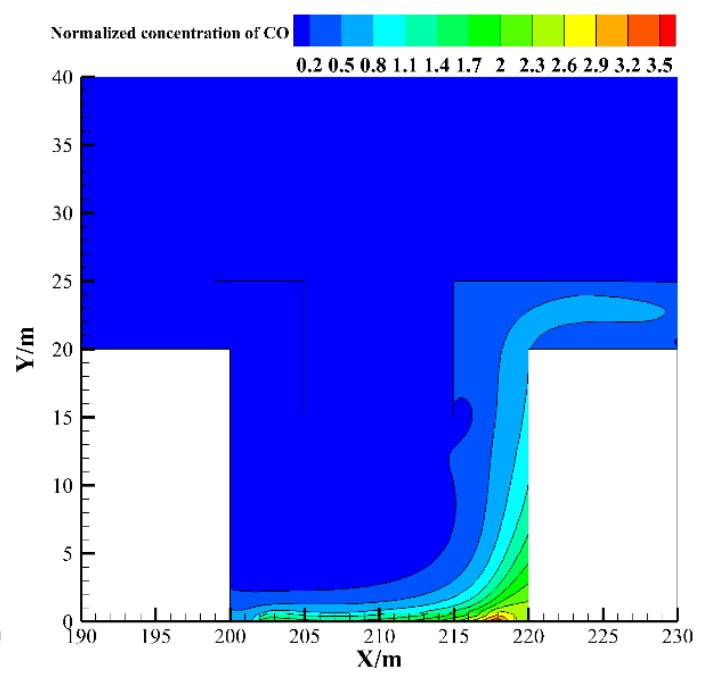

(d) 


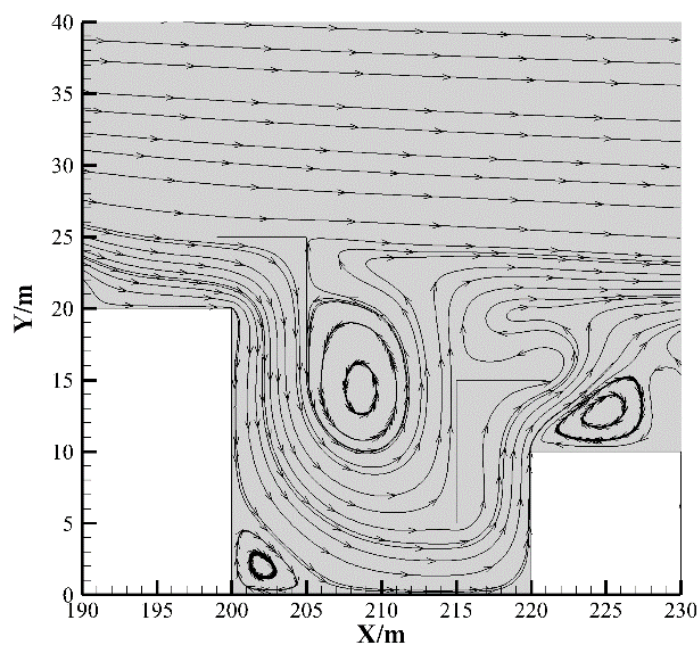

(e)

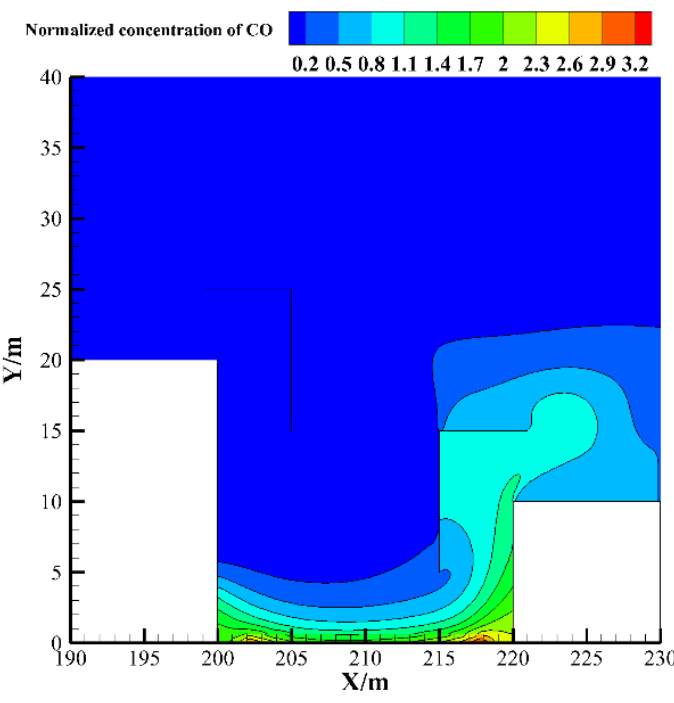

(f)

Fig. 9 Flow fields and dimensionless concentrations of $\mathrm{CO}$ in three street canyon types equipped with wind catchers. (a)and(b), step-up street canyon; (c)and(d), symmetrical street canyon;

(e)and(f), step-down street canyon.

The changes in the synergy angle, Sherwood number, and average CO concentration were determined to quantify the impact of the wind catcher on the pollutant dispersion in the street canyon. In Figure 10, the synergy angle changed in the three different street canyon types after the wind catcher was installed. When the modulus of the two vectors of velocity and concentration gradient remained constant, it was observed that the smaller the synergy angle, the better the synergy was. (1) In the step-up street canyon(fig10(a) and fig10(b)), the synergy angle of the central area of the street canyon was significantly lower, and the area with an angle smaller than $20^{\circ}$ increased by nearly three times. The synergy in the street canyon was significantly improved. (2) In the symmetrical street canyon (fig10(c) and fig10(d)), the left half of the street canyon had a smaller synergy angle, and the middle area of the two wind catchers had a larger synergy angle. Overall, the synergy of the street canyon area was improved. (3) In the step-down street canyon (fig10(e) and fig10(f)), the area with a larger coordination angle was significantly larger. In summary, the wind catcher had a significant effect on the synergy of the vector field, and the most significant effect occurred in the step-up street canyon.
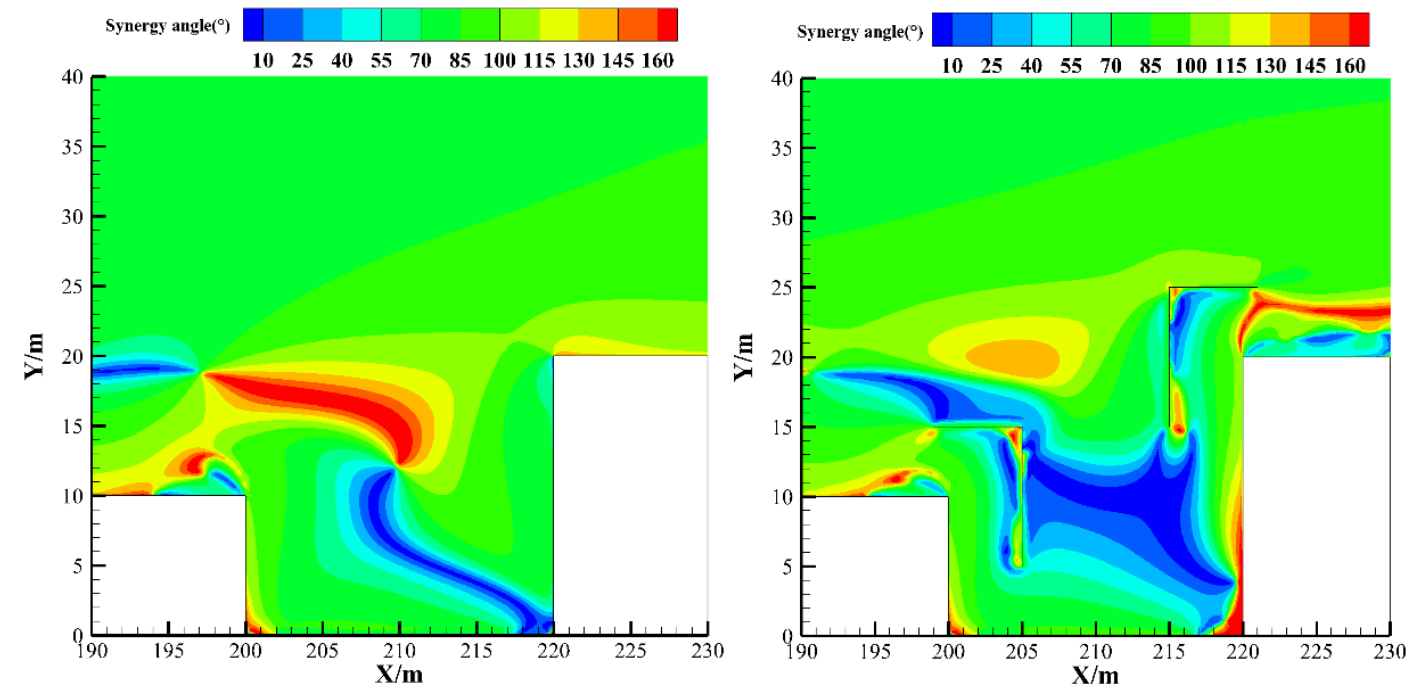
(a)

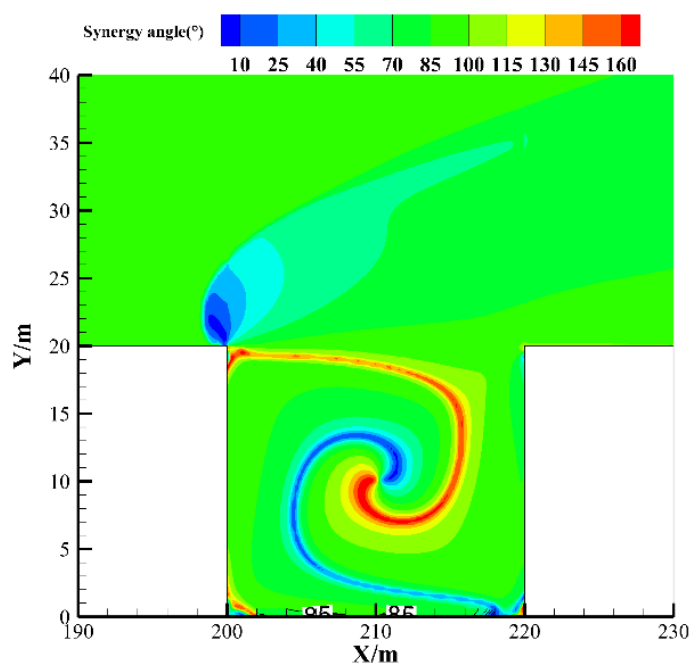

(c)

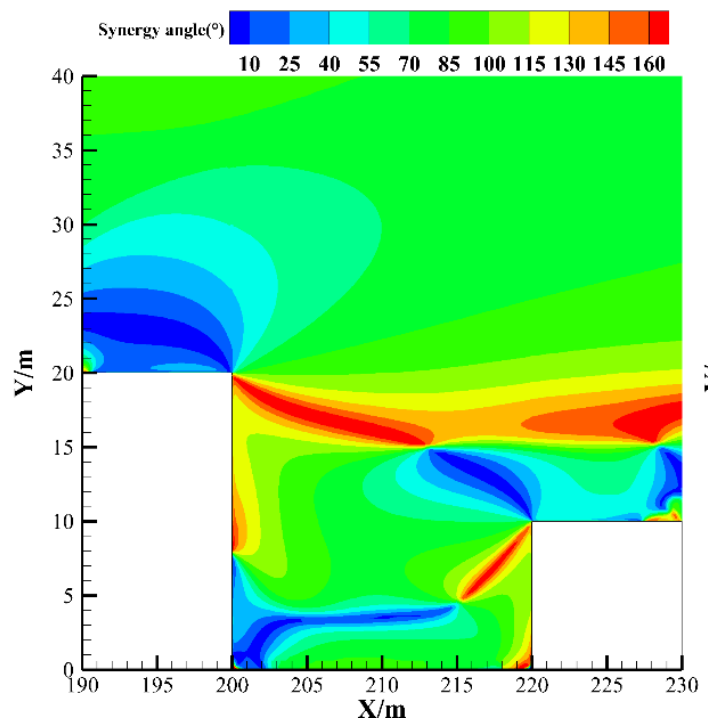

(e) (b)

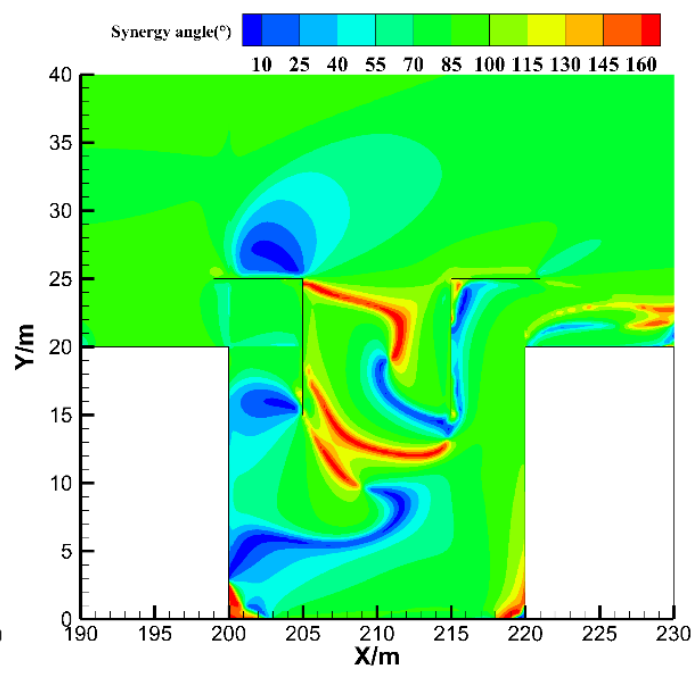

(d)

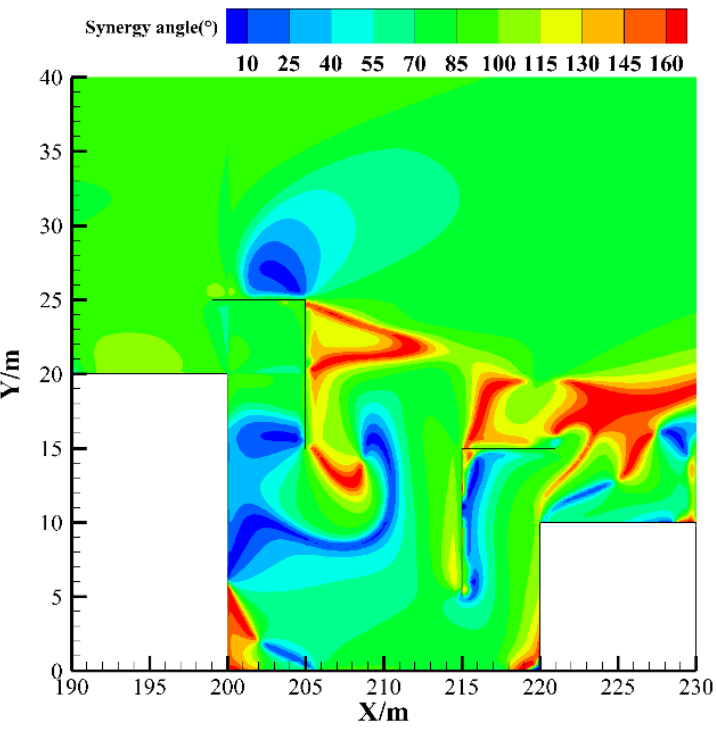

(f)

Fig. 10 Comparison of synergy angles after installment of the wind catchers in the street canyon. (a)and(b), step-up street canyon; (c)and(d), symmetrical street canyon; (e)and(f), step-down street canyon.

The wind catcher changes the size of the flow field in the street canyon, i.e., the Reynolds number changes. Figure 11 shows the effects of the wind catcher on the Sherwood number and the average $\mathrm{CO}$ concentration in the street canyons. The results show that the wind catcher increased the Sherwood number in all three types of street canyons, and the largest effect occurred in the stepdown street canyon. The Sherwood number increased by nearly four times in the step-down street canyon and by nearly two times in the other two street canyons. The average $\mathrm{CO}$ concentration decreased in the symmetrical street canyon and the step-down street canyon, and the rate of decrease was similar to the rate of increase in the Sherwood number. In the step-up street canyon, the average concentration of pollutants in the street canyon increased slightly after the wind catcher was installed. The results demonstrate that the field synergy of the step-up street canyon was significantly improved, but the wind catcher changed the flow in the street canyon. It is not possible 
to use the same Reynolds number for the entire street canyon and obtain a representative Sherwood number of the full flow field. However, there is not much difference between the average CO concentrations of the two cases, and it is believed that the Sherwood number of the convective mass transfer field synergy theory can be used to evaluate the dispersion of pollutants in street canyons.

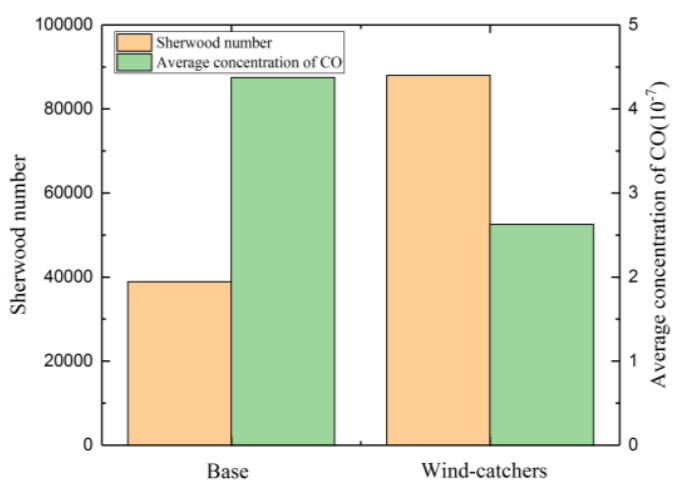

(a)

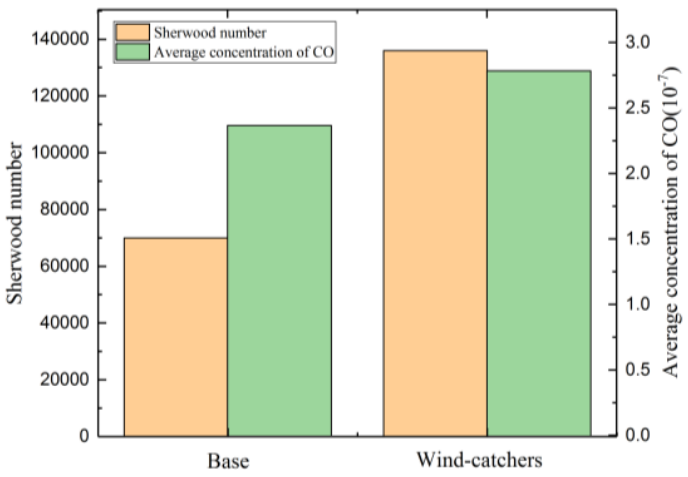

(b)

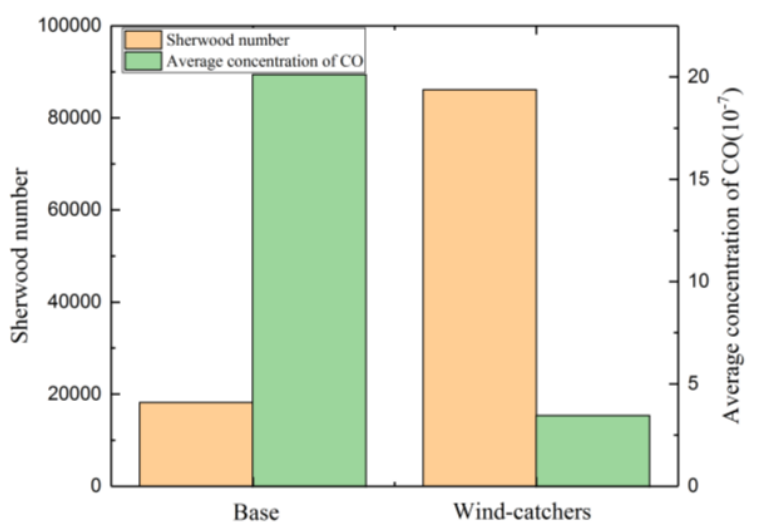

(c)

Fig. 11 The Sherwood number and the average CO concentration in the street canyons after adding the wind catcher.(a) symmetrical street canyon;(b)step-up street canyon;(c)step-down street canyon

\subsection{Influence of ambient wind velocity}

As reported in ${ }^{[47]}$, deterioration in air quality in street canyons usually occurs when the airflow is perpendicular or parallel to the street canyon. We investigated the case where the ambient wind direction is perpendicular to the street canyon to simplify the model to two dimensions and save computing resources because it is known that there is an effect of the wind speed on the flow structure and pollutant dispersion in street canyons. Five types of ambient wind speed are investigated, i.e., $1 \mathrm{~m} / \mathrm{s}, 2 \mathrm{~m} / \mathrm{s}, 3 \mathrm{~m} / \mathrm{s}, 4 \mathrm{~m} / \mathrm{s}$, and $5 \mathrm{~m} / \mathrm{s}$ to represent very light wind, light wind, medium wind, high wind, and very high wind.

In this case, the wind speed and the Reynolds number are different. The field synergy number does not represent the effect of the convective mass transfer and dispersion. Therefore, the changes in the Sherwood number and average CO concentration for different wind speeds in different street canyon types were determined. Figure 12 shows that as the wind speed increases, the average pollutant concentrations decrease in all canyon types. The step-up street canyon has the largest Sherwood number, indicating the structural advantages of the step-up street canyon. Both the 
increase in the Reynolds number and the increase in the field synergy number cause an increase in the Sherwood number. The result shows that the wind speed has different effects on the synergy of the two vectors of wind speed and concentration gradient in the three street canyon types. The ranking of the average $\mathrm{CO}$ concentration from lowest to highest is step-up street canyon $<$ symmetric street canyon $<$ step-down street canyon. Figure 12 (b) shows that the step-down street canyon has the largest rate of decrease in the average $\mathrm{CO}$ concentration. The reason is that the step-down street canyon has the most even distribution of pollutants, and as the wind speed changes, the area of convective mass transfer and dispersion increases, resulting in a rapid decrease in the average $\mathrm{CO}$ concentration in the entire street canyon. In addition, as the wind speed increases, the rate of decrease in the average $\mathrm{CO}$ concentration decreases.

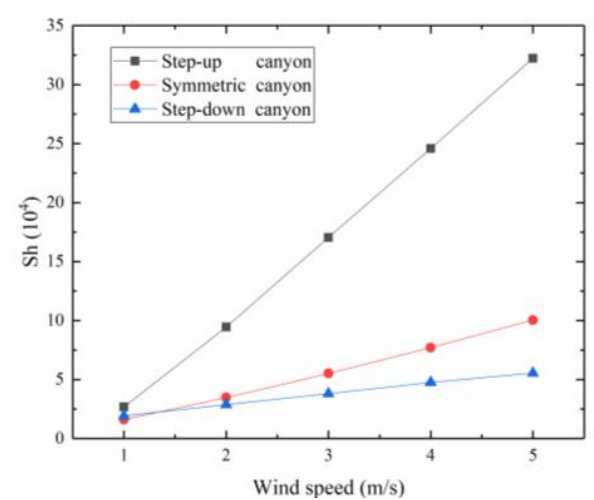

(a)

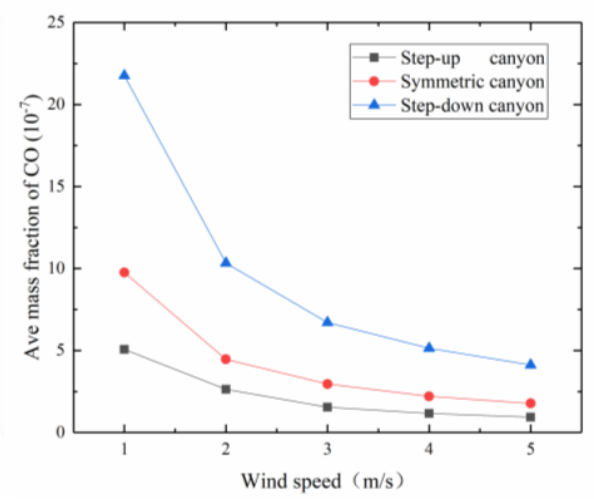

(b)

Fig. 12 Sherwood number and average $\mathrm{CO}$ concentration at different wind velocities in different street canyon types.(a) Sherwood number;(b) average CO concentration

\section{Conclusions}

Based on the similarity between convective mass transfer and convective heat transfer, the field synergy theory is extended to the convective mass transfer analysis of pollutant dispersion in street canyons. Accroding to this theory, Sherwood number was used to evaluate the impact of wind catchers on improving the ambient air quality of street canyon. The changes in the synergy angle explained the reason for this effect. Sherwood number was also used to quantitatively measure the pollutant diffusion in step-up and step-down street canyons. Since Sherwood number is positively correlated with Reynolds number, field synergy number is used to measure the effect of wind speed changes on the dilution of pollutants in the street canyon. The following conclusions can be drawn:

At the same wind speed, the pollutant dispersion performance differed for the different street canyon types. In the step-up street canyon, the pollutants were dispersed to the adjacent street canyon. The pollutant concentration in the step-down street canyon was significantly higher than that in the other two street canyons. The addition of wind catchers to the upper part of buildings significantly improved the synergy between the velocity and the concentration gradient vector field in the street canyon. The pollutants were significantly reduced in the symmetrical and step-down street canyon. In the step-down street canyon, the Sherwood number increased about 4 times, and the average concentration in the street canyon dropped to about a quarter. The effect of the wind catcher on the dispersion of pollutants was different in the three street canyons, and the performance ranking was as follows: step-down street canyon $>$ symmetrical street canyon $>$ set-up street canyon. 
Therefore, the addition of wind catchers in the step-down street canyon provides the best performance.

When the wind direction was perpendicular to the street canyon, the larger the wind speed, the larger the Sherwood number was, and the greater the dispersion of pollutants in the street canyon was. However, the effect of the wind speed on the dispersion of pollutants differed for different street canyon types. The pollutant dispersion performance was as follows: step-up street canyon $>$ symmetrical street canyon $>$ step-down street canyon.

\section{Acknowledgments}

This research was supported by the National Natural Science Foundation of China (Grant No. 51778511), the European Commission H2020 Marie S Curie Research and Innovation Staff Exchange (RISE) award (Grant No. 871998), Hubei Provincial Natural Science Foundation of China (Grant No. 2018CFA029), and Key Project of ESI Discipline Development of Wuhan University of Technology (Grant No. 2017001).

\section{References}

[1] Yazid AWM, Sidik NaC, Salim SM, et al. A review on the flow structure and pollutant dispersion in urban street canyons for urban planning strategies. Simulation, 2014,90(8): 892-916.

[2] Lateb, M, Meroney, RN, Yataghene, M, Fellouah, H, Saleh, F, Boufadel, M C, On the use of numerical modelling for near-field pollutant dispersion in urban environments - a review. Environmental Pollution, 2016, 208: 271-283.

[3] Zhang, YW, Gu, Z, Chuck, WY, Review on numerical simulation of airflow and pollutant dispersion in urban street canyons under natural background wind conditions. Aerosol \& Air Quality Research, 2018, 18: 780-789.

[4] Blocken, B, Tominaga, Y, Stathopoulos, T, CFD simulation of micro-scale pollutant dispersion in the built environment. Building \& Environment, 2013,64:225-230.

[5] Salim, SM, Buccolieri, R, Chan, A, Di Sabatino, S, Numerical simulation of atmospheric pollutant dispersion in an urban street canyon: comparison between RANS and LES. Journal of Wind Engineering \& Industrial Aerodynamics, 2011, 99(2-3):103-113.

[8] MacDonald, RW, Griffths, RF, Hall, DJ, A comparison of results from scaled field and wind tunnel modelling of dispersion in arrays of obstacles. Atmospheric Environment, 1998,32(22): 3845-3862.

[7] Chang, $\mathrm{CH}$, Meroney, $\mathrm{RN}$, Concentration and flow distributions in urban street canyons: wind tunnel and computational data. Journal of Wind Engineering \& Industrial Aerodynamics, 2003,91:1141-1154.

[8] Kastner-Klein, P, R Berkowicz, and R Britter, The influence of street architecture on flow and dispersion in street canyons. Meteorology \& Atmospheric Physics, 2004,87(1-3): 121-131.

[9] Xie, X, H Zhen, and JS Wang, Impact of building configuration on air quality in street canyon. Atmospheric Environment, 2005,39(25): 4519-4530.

[10] Kim JJ and Baik JJ. A numerical study of the effects of ambient wind direction on flow and dispersion in urban street canyons using the RNG k-e turbulence model, Atmospheric 
Environment ,2004, 38: 3039-3048.

[11] Soulhac L, Perkins RJ and Salizzoni P. Flow in a street canyon for any external wind direction. Boundary Layer Meteorol, 2008, 126: 365-388.

[12] Liu CH, Leung DYC and Barth MC. On the prediction of air and pollutant exchange rates in street canyons of different aspect ratios using large-eddy simulation. Atmospheric Environment ,2005, 39: 1567-1574.

[13] Gu ZL, Zhang YW, Cheng Y, et al. Effect of uneven building layout on air flow and pollutant dispersion in non-uniform street canyons. Building \& Environment, 2011,46: 2657-2665.

[14] Baker J, Walker HI and Cai X. A study of the dispersion and transport of reactive pollutants in and above street canyons - a large eddy simulation. Atmospheric Environment, 2004, 38:68836892 .

[15] Merah, A, and A. Noureddine, Reactive Pollutants Dispersion Modeling in a Street Canyon. International Journal of Applied Mechanics and Engineering, 2019,24(1): 91-103.

[16] Jian Hang,Zhiwen Luo,Xuemei Wang,Lejian He,Baomin Wang,Wei Zhu, The influence of street layouts and viaduct settings on daily carbon monoxide exposure and intake fraction in idealized urban canyons. Environmental Pollution, 2017, 220(Pt A): 72-86.

[17] Chenrui Hao,Xiaomin Xie,Yue Huang,Zhen Huang, Study on influence of viaduct and noise barriers on the particulate matter dispersion in street canyons by CFD modeling. Atmospheric Pollution Research, 2019,10(6):1723-1735.

[18] Uehara K, Murakami S, Oikawa S, et al. Wind tunnel experiments on how thermal stratification affects flow in and above urban street canyons. Atmospheric Environment ,2000, 34:1553-1562.

[19] Xie X, Huang Z, Wang J, et al. The impact of solar radiation and street layout on pollutant dispersion in street canyon. Building \& Environment ,2005, 40: 201-212.

[20] Nowak DJ, Crane DE and Stevens JC. Air pollution removal by urban trees and shrubs in the United States.Urban For Urban Greening ,2006, 4: 115-123.

[21] Tallis M, Taylor G, Sinnett D, et al. Estimating the removal of atmospheric particulate pollution by the urban tree canopy of London, under current and future environments. Landscape Urban Plann, 2011,103: 129-138.

[22] Sharples, S, Bensalem, R, Airflow in courtyard and atrium buildings in the urban environment: A wind tunnel study. Solar Energy, 2001, 70: 237-244.

[23] Montazeri, H, Experimental and numerical study on natural ventilation performance of various multi-opening wind catchers. Building \& Environment, 2011, 46: 370-378.

[24] Montazeri, H, Azizian, R. Experimental study on natural ventilation performance of one-sided wind catcher. Building \& Environment, 2008, 43:2193-2202.

[25] Dehghan, A, Esfeh, MK, Manshadi, MD, Natural ventilation characteristics of one-sided wind catchers: Experimental and analytical evaluation. Energy \& Buildings, 2013, 61:366-377.

[26] Hosseini S H, Shokry E, Hosseini A J A, et al. Evaluation of airflow and thermal comfort in buildings ventilated with wind catchers: Simulation of conditions in Yazd City, Iran. Energy for Sustainable Development, 2016, 35(DEC.):7-24.

[27] Haghighi A P , Pakdel S H , Jafari A, A study of a wind catcher assisted adsorption cooling channel for natural cooling of a 2-storey building. Energy, 2016, 102(May 1):118-138.

[28] Calautit J , Aquino A, O'Connor D , et al. Indoor environmental quality (IEQ) analysis of a low energy wind catcher with horizontally-arranged heat transfer devices. Energy Procedia, 2017, 142:2095-2101. 
[29] Montazeri, H, and F. Montazeri, CFD simulation of cross-ventilation in buildings using rooftop wind-catchers: Impact of outlet openings. Renewable Energy, 2017,118:502-520.

[30] Lup, C, N. Negin, and N. Leslie, Pedestrian-Level Urban Wind Flow Enhancement with Wind Catchers. Atmosphere, 2017, 8(9): 159.

[31] Zhang K, Chen G, Wang X, et al. Numerical evaluations of urban design technique to reduce vehicular personal intake fraction in deep street canyons. Science of the Total Environment, 2019, 653:968-994.

[32] Zhengtong Li, Tianhao Shi, Yongjia Wu, et al. Effect of traffic tidal flow on pollutant dispersion in various street canyons and corresponding mitigation strategies. Energy and Built Environment, 2020, 1(3):242-253.

[33] Guo, ZY, DY Li, and BX Wang, A novel concept for convective heat transfer enhancement. International Journal of Heat \& Mass Transfer,1998,41(14): 2221-2225.

[34] Han, G, B Hua, and Y Wei, A new approach of transfer process enhancement-field synergy. Nature Magazine, 2002, 7(1).

[35] Wei, L, ZC, Liu, and SY, Huang, Physical quantity synergy in the field of turbulent heat transfer and its analysis for heat transfer enhancement. Chinese Science Bulletin, 2010, 55(23): 2589-2597.

[36] Zeng, $M$ and $W$, Tao, Numerical verification of the field synergy principle for turbulent flow. Journal of Enhanced Heat Transfer, 2004, 11(4): 453-460.

[37] Liu, W, ZC, Liu, and ZY, Guo, Physical quantity synergy in laminar flow field of convective heat transfer and analysis of heat transfer enhancement. Chinese Science Bulletin, 2009,54(19): 3579-3586.

[38] Chen, Q, J, Ren, and Z, Guo, Field synergy analysis and optimization of decontamination ventilation designs. International Journal of Heat \& Mass Transfer, 2008, 51(3-4): 873-881.

[39] Blocken B, Stathopoulos T, Saathoff P, et al. Numerical evaluation of pollutant dispersion in the built environment: Comparisons between models and experiments. Journal of Wind Engineering \& Industrial Aerodynamics,2008,96(10-11):1817-1831.

[40] Nazridoust, K and G, Ahmadi, Airflow and pollutant transport in street canyons. Journal of Wind Engineering \& Industrial Aerodynamics, 2006, 94(6): 491-522.

[41] Assimakopoulos, VD, HM, ApSimon, and N, Moussiopoulos, A numerical study of atmospheric pollutant dispersion in different two-dimensional street canyon configurations.

Atmospheric Environment, 2003, 37(29):4037-4049.

[42] Leung K K, Liu C-H, Wong C C C, et al. On the study of ventilation and pollutant removal over idealized two-dimensional urban street canyons. Building Simulation, 2012, 5(4): 359-369.

[43] Hefny M M, Ooka R. CFD analysis of pollutant dispersion around buildings: Effect of cell geometry. Building and Environment, 2009, 44(8): 1699-1706.

[44] Michioka T, Sato A, Takimoto H, et al. Large-Eddy Simulation for the Mechanism of Pollutant Removal from a Two-Dimensional Street Canyon. Boundary-Layer Meteorology, 2011, 138(2): 195-213.

[45] Pavageau, M and M, Schatzmann, Wind tunnel measurements of concentration fluctuations in an urban street canyon. Atmospheric Environment, 1999, 33(24-25): 3961-3971.

[46] Montazeri, $\mathrm{H}$ and R, Azizian, Experimental study on natural ventilation performance of onesided wind catcher. Building \& Environment, 2008, 43(12): 2193-2202.

[47] Blocken B, Stathopoulos T, Carmeliet J, et al. Application of computational fluid dynamics in building performance simulation for the outdoor environment: An overview. Journal of Building 
Performance Simulation, 2011,4(2): 157-184. 